\title{
Anakinra for Treatment-Resistant Kawasaki Disease: Evidence from a Literature Review
}

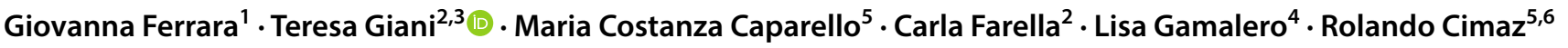

Published online: 3 September 2020

(c) Springer Nature Switzerland AG 2020

\begin{abstract}
Kawasaki disease (KD) is one of the most common vasculitides of childhood and the main cause of acquired heart disease in developed countries. Intravenous immunoglobulin (IVIG) in association with aspirin represents the main treatment for KD. However, $10-20 \%$ of patients fail to respond to standard treatment and have an increased risk of cardiac complications. There is currently no accepted protocol for treatment of resistant cases. Several authors highlighted the role of interleukin-1 (IL-1) as a mediator of inflammation in KD and suggested the possibility of using IL-1 or its receptor as a target of therapy. The use of IL-1 inhibitors in patients with KD has been reported in the scientific literature, but data are largely limited to individual case reports and small case series. We summarized the scientific literature related to the use of anakinra, analyzing preclinical and clinical data. Thirty-eight patients have been described so far, most of them with KD-related complications. Twenty-two were described in case reports and case series, while 16 were patients from the completed KAWAKINRA phase IIa study. Almost all patients received clinical benefit, and no relevant side effects were noted. Based on this evidence, in our opinion, anakinra may be considered as an option after the failure of the first IVIG infusion, especially in patients with coronary involvement.
\end{abstract}

\section{Key Points}

Interleukin-1 (IL-1) plays a key role in the pathogenesis of Kawasaki disease (KD), especially in the development of coronary artery aneurysms.

Based on current evidence, derived from case reports and one open-label, phase II study, the IL-1 inhibitor anakinra is a fast-acting, effective, and reasonably safe drug.

Anakinra may be considered as an option to treat KD resistant to a first intravenous immunoglobulin (IVIG) dose, especially in the presence of severe coronary involvement.

Teresa Giani

teresa.giani@mail.com

1 ASL Toscana Centro, Florence, Italy

2 AOU Meyer, Florence, Italy

3 Department of Medical Biotechnology, University of Siena, Siena, Italy

4 University of Udine, Udine, Italy

5 ASST G-Pini, Milan, Italy

6 Department of Clinical Sciences and Community Health and RECAP-RD, University of Milan, Milan, Italy

\section{Introduction}

Kawasaki disease (KD) is an acute medium vessel vasculitis of childhood, typically involving coronary arteries, and represents in this age group the first cause of acquired heart disease in developed countries. Classically, KD is diagnosed in the presence of a high fever lasting for at least 5 days associated with at least four of the five principal features: changes in the peripheral extremities, cervical lymphadenopathy, bilateral non-exudative conjunctivitis, changes in the oral cavity, and polymorphous exanthema [1]. In the presence of fever and four or more principal clinical features, the diagnosis of complete KD can be made; experienced clinicians who have treated many patients with KD may also establish the diagnosis without waiting for the full clinical picture and even with $<4$ days of fever [1]. The diagnosis is clinical, and there are no confirmatory laboratory or imaging investigations. If coronary artery abnormalities are detected, the diagnosis of $\mathrm{KD}$ is considered confirmed also in those cases that lack the full clinical features of classic KD and that would otherwise be considered as incomplete KD cases [1]. Intravenous immunoglobulin (IVIG) in association with aspirin represents the main treatment for $\mathrm{KD}$, and administration of these treatments within the first 10 days following fever onset has been associated with a fivefold reduction in the risk of coronary artery aneurysms (CAA) [1]. However, 10-20\% of patients do not 
respond to standard treatment and have an increased risk of cardiac complications and death [2].

The current evidence to guide treatment of resistant KD is of moderate to low quality [2], which is unfortunate given the potential severity of adverse outcomes of both KD itself and the treatments, and the cost of some proposed agents. The latest guidelines of the American Heart Association [1] suggest that reasonable therapy for resistant KD includes a second dose of IVIG or a short course of high-dose steroids or infliximab. The guidelines also mention the anti-interleukin-1 (anti-IL-1) anakinra among the different drugs that can be used in refractory forms, but leave the choice of the drug to individual physicians. Interleukin-1 (IL-1) has been shown to play a key role in the development of CAA, leading to a potential use of IL-1 blockade in patients with KD [3]. Our aim was to review the literature in order to report the scientific evidence coming from both experimental and clinical studies that support the use of anakinra in refractory KD.

A careful search using PubMed and the Cochrane Library for human studies published in English up to the present time and the platform ClinicalTrials.gov for ongoing trials was conducted. The key words used in the search were "Kawasaki" and "anakinra" (Kawasaki [All Fields] AND "interleukin 1 receptor antagonist protein"[MeSH Terms] OR "interleukin 1 receptor antagonist protein"[All Fields] OR “anakinra”[All Fields]).

\section{Anti-IL-1 Drugs}

In the last few decades, biological disease-modifying antirheumatic drugs have been developed to interfere with IL-1 signaling. The activation of IL- 1 signaling requires the combination of IL-1 with type I IL-1 receptor and IL-1 receptor accessory protein, which ultimately activates the transcription factor nuclear factor kappa light-chain enhancer of activated B cells (NF- $\kappa \mathrm{B}$ ) [4]. The three drugs currently available either block the IL-1 binding to the IL-1 receptor (anakinra) or bind directly to IL-1 (rilonacept and canakinumab).

Anakinra is an IL-1 receptor antagonist, and was approved by the US Food and Drug Administration (FDA) for the treatment of rheumatoid arthritis in 2001 and cryopyrin-associated periodic syndrome in 2013 [5]. It is a non-glycosylated homolog of human IL-1 receptor antagonist (IL-1Ra), manufactured from cultures of genetically modified Escherichia coli using recombinant DNA technology, and differs from the native human IL-1RA in an extra methionine residue on its amino terminus [5]. Anakinra, unlike canakinumab and rilonacept, acts as a competitive inhibitor of both IL- $1 \alpha$ and IL- $1 \beta$, blocking signal transduction by IL-1 type I receptor; of note, it has been demonstrated that IL- $1 \alpha$ also has a major role in coronary lesions [6].

A big advantage of this drug over the other anti-IL-1 agents is the rapid onset of action and the short half-life of 4-6 $\mathrm{h}$; these aspects confer the advantages of being able to quickly test the response and limit the effects in the case of a serious adverse event [7]. This makes the drug an extremely manageable treatment in clinical practice. On the contrary, for long-term illnesses, the daily subcutaneous injections represent a major disadvantage.

Anakinra is now used in numerous diseases such as hereditary autoinflammatory syndromes, and was recently approved for systemic onset juvenile idiopathic arthritis and Still's disease. In children weighing less than $50 \mathrm{~kg}$, the starting dosage is $1-2 \mathrm{mg} / \mathrm{kg} /$ day; patients weighing $50 \mathrm{~kg}$ or more are dosed with $100 \mathrm{mg} /$ day. In children with inadequate response, the dosage can be escalated up to $4 \mathrm{mg} / \mathrm{kg} / \mathrm{day}$.

Rilonacept is a fully human dimeric fusion protein consisting of both IL-1 receptor components required for IL-1 signaling (IL-1 type I receptor and IL-1 receptor accessory protein) combined with the Fc portion of human immunoglobulin G1. It acts by blocking both IL- $1 \alpha$ and IL- $1 \beta$. The drug was first approved by the US FDA in 2008 for the treatment of cryopyrin-associated periodic syndrome. It has a half-life of 8 days, and is administered by subcutaneous weekly injections (160 mg/week) [8].

Canakinumab is a recombinant, human monoclonal, highly selective anti-IL- $1 \beta$ antibody, able to prevent the binding of endogenous IL- $1 \beta$ to its cognate receptor. The drug was first approved by the US FDA in June 2009 for the treatment of familial cold autoinflammatory and Muckle-Wells syndromes. Serum clearance of canakinumab is slow after subcutaneous injection, with a long half-life of 21-30 days. It therefore offers the advantage of monthly injections [9].

\section{Experimental Data}

The first studies demonstrating high levels of IL-1 in patients with KD during the acute phase date back to more than 30 years ago [10].

A more recent study confirms this finding, showing significantly increased plasma levels of IL- $1 \beta, \mathrm{IL}-18$, and their antagonists (IL-1RA and IL-18BP) in acute KD patients compared with age-matched control patients with viral or bacterial infections [11].

In the last decade, several studies demonstrated the key role of IL-1 in the pathogenesis of KD.

Weng et al. enrolled 156 children with KD (136 responders and 20 non-responders to IVIG) and showed that polymorphisms in the genes coding for IL-1 (-31 CC and -511 
TT) were associated with a greater risk of resistance to IVIG treatment [12].

Similar results were obtained by another group, who evaluated IL-1-related gene transcripts in patients with IVIG-resistant KD. Again, considering $20 \mathrm{KD}$ patients (12 responders and eight resistant) and nine controls, the authors confirmed a significant abundance of transcripts in IVIG-resistant patients, demonstrating an increased expression of the coding genes for proteins implicated in the IL-1 pathway. The authors therefore highlighted the role of IL-1 as a mediator of inflammation in KD and suggested the possibility of using IL-1 or its receptor as a target of therapy [13].

The same conclusions came from a study comparing the gene expression of 146 patients with KD in the acute and subacute phase with that of subjects suffering from other acute infectious diseases and healthy controls [14].

The efficacy of anti-IL-1 treatment was studied by Lee et al. in a mouse model of coronary arteritis developed using intraperitoneal injection of Lactobacillus casei cellwall extract (LCCWE). The mice developed a focal, localized coronary arteritis that histopathologically mimics the coronary artery lesions found in human KD [15]. In a first experiment, mice lacking caspase-1 (Caspase-1 -/-), an enzyme of the inflammasome that activates IL-1, were unable to trigger the inflammatory response to LCCWE and did not develop coronary arteritis; similar results were obtained in mice without the IL-1 receptor (IL-1R -/-). In a second experiment, mice treated with anakinra daily, from the day before exposure to LCCWE until day 5 , and then sacrificed on day 7 for anatomical search of coronary damage, showed a significantly lower incidence of vasculitis than controls. The same experiment was also conducted with a tumor necrosis factor inhibitor, infliximab. Also in this case, there was a reduced incidence of coronary lesions compared to controls, but not of myocarditis. In addition, treatment with anakinra showed greater efficacy than infliximab (protection in $8 / 9$ vs. 5/9) [16].

In mice models of severe forms of $\mathrm{KD}$, the same group evaluated IL-1 blockade in preventing and reducing systemic vascular inflammation, in particular, of the abdominal vessels (renal arteries and aorta) [17].

A recent study by the same authors showed how blocking IL-1 also prevents the long-term consequences of KD. In particular, in order to verify the development of ventricular dysfunction and to study the volume of the left ventricle, the authors performed serial echocardiograms on LCCWEexposed mice, up to 43 days after the injection of LCCWE. Three weeks following the injection, mice exposed to the lysate began to show a reduced ejection fraction compared to controls. In those who had been pre-treated with anakinra, however, these changes did not occur. The authors concluded on the effectiveness of anakinra in preventing not only acute damage, but also long-term sequelae, and proposed a wider use of the drug, not only for difficult cases [18].

Moreover, it was recently shown that IL-1 signaling in intestinal epithelial cells increased intestinal permeability, as deleting IL-1R specifically in those cells not only prevented increased permeability, but also reduced heart inflammation and aortic aneurysm development in LCCWE-injected KD mice [19].

\section{Clinical Data}

\subsection{Case Reports}

Several case reports of KD patients treated with anakinra have been published (nine single cases, a series of two, and a series of 11); their main characteristics are summarized in Table 1.

The first report on the use of anti-IL-1 in KD dates back to 2012 [20] and described a 2-year-old boy with classic KD who developed myocarditis with reduction of the ejection fraction to $20 \%$, without coronary artery inflammation, 2 days after the first dose of IVIG. Despite a second dose of IVIG and three boluses of methylprednisolone, echocardiography showed a dilatation of the left descending coronary and right coronary arteries. On the 14th day, the patient was intubated and supported with extracorporeal membrane oxygenation (ECMO) for respiratory distress and cardiogenic shock. On the 18th day, anakinra $1 \mathrm{mg} / \mathrm{kg}$ was started, with prompt resolution of the fever and a significant clinical improvement. Three days after the withdrawal of anakinra (on the 29th day), fever reappeared, IVIG therapy was readministered, and methylprednisolone was resumed. On the 53rd day, echocardiography showed a giant aneurysm of one coronary artery. Anakinra was re-administered for 6 weeks, in combination with aspirin and anticoagulants to prevent thrombosis. After 6 months from disease onset, echocardiography showed a complete normalization of the cardiovascular findings. In this case, the close correlation between the rapid relapse of the disease with the drug withdrawal and the immediate clinical recovery on its reintroduction support the effectiveness of the anti-IL-1.

Shafferman et al. [21] in 2014 reported on an 11-weekold infant with classic KD who developed respiratory failure. The patient was intubated, and a lumbar puncture was performed, showing a sterile pleiocytosis. Laboratory data revealed anemia, thrombocytopenia, and elevated inflammatory markers. Echocardiography showed a slight dilatation of the coronary arteries, and abdominal ultrasound showed hydrops of the gallbladder. Therapy with IVIG and aspirin was started, but with a sudden increase in ferritin and a further decrease in platelet count, with suspicion of macrophage activation syndrome (MAS), methylprednisolone 
Table 1 Main details of case reports reporting the use of anakinra in treatment-resistant Kawasaki disease

\begin{tabular}{|c|c|c|c|c|c|}
\hline Reference & $\begin{array}{l}\text { Number of patients } \\
\text { treated with anak- } \\
\text { inra }\end{array}$ & Previous treatment & Type of KD & Reason for using anakinra & $\begin{array}{l}\text { Doses and duration of anakinra } \\
\text { treatment }\end{array}$ \\
\hline$[20]$ & 1 & 2 IVIG, 3 steroid boluses & Complete & KD shock syndrome & $\begin{array}{l}1 \mathrm{mg} / \mathrm{kg} / \mathrm{day} \text { for } 11 \text { days and } \\
\text { then for } 6 \text { weeks }\end{array}$ \\
\hline$[21]$ & 1 & 1 IVIG, 1 steroid bolus & Complete & $\begin{array}{l}\text { Macrophage activation } \\
\text { syndrome }\end{array}$ & $\begin{array}{l}3 \mathrm{mg} / \mathrm{kg} / \text { day for } 3 \text { days then } \\
8 \mathrm{mg} / \mathrm{kg} / \text { day in } 2 \text { doses for } \\
5 \text { months }\end{array}$ \\
\hline$[22]$ & 1 & 2 IVIG, 4 steroid boluses & Complete & $\begin{array}{l}\text { Persistent fever and labora- } \\
\text { tory abnormalities }\end{array}$ & $2 \mathrm{mg} / \mathrm{kg} /$ day for 2 weeks \\
\hline$[23]$ & 1 & 2 IVIG, 3 steroid boluses & Complete & $\begin{array}{l}\text { Worsening of coronary } \\
\text { aneurysms }\end{array}$ & $\begin{array}{l}6 \mathrm{mg} / \mathrm{kg} / \mathrm{day} \text { for } 10 \mathrm{weeks} \text {, } \\
\text { Tapered to } 6 \mathrm{mg} / \mathrm{kg} / 2 \text { days } \\
\text { for } 4 \text { weeks, tapered to } 6 \mathrm{mg} / \\
\mathrm{kg} / 3 \text { days for } 4 \text { weeks }\end{array}$ \\
\hline$[24]$ & 1 & $1 \mathrm{IVIG}$ & Complete & $\begin{array}{l}\text { Coronary aneurysms and } \\
\text { increased proBNP levels }\end{array}$ & $100 \mathrm{mg} /$ day for 4 weeks \\
\hline$[25]$ & 11 & Al least two & $\begin{array}{l}9 \text { complete } \\
2 \text { incomplete }\end{array}$ & $\begin{array}{l}\text { Persistent fever }(8 / 11), \\
\text { dilatation of coronary } \\
\text { arteries }(7 / 11), \text { poor clinical } \\
\text { conditions }(2 / 11), \text { labora- } \\
\text { tory abnormalities }(6 / 11), \\
\text { myocarditis with KD shock } \\
\text { syndrome }(1 / 11)\end{array}$ & $\begin{array}{l}\text { Range } 2-8 \mathrm{mg} / \mathrm{kg} / \text { day for } \\
\text { 6-81 days }\end{array}$ \\
\hline$[26]$ & 1 & Several, not specified & NA & Giant aneurysm & NA \\
\hline$[27]$ & 2 & Several, not specified & NA & KD shock syndrome & NA \\
\hline$[28]$ & 1 & $\begin{array}{l}2 \text { IVIG, } 1 \text { infliximab, } 3 \text { ster- } \\
\text { oid boluses }\end{array}$ & Incomplete & $\begin{array}{l}\text { Macrophage activation } \\
\text { syndrome }\end{array}$ & $\begin{array}{l}5 \mathrm{mg} / \mathrm{kg} / \mathrm{day} \text { for } 1 \text { day and then } \\
10 \mathrm{mg} / \mathrm{kg} / \text { day tapering in } \\
6 \text { months }\end{array}$ \\
\hline [29] & 1 & $1 \mathrm{IVIG}$ & Incomplete & Coronary aneurysms & $4 \mathrm{mg} / \mathrm{kg} /$ day for 2 months \\
\hline$[30]$ & 1 & $\begin{array}{l}2 \text { IVIG, } 3 \text { steroid boluses, } 1 \\
\text { infliximab }\end{array}$ & Complete & Giant aneurysms & $6 \mathrm{mg} / \mathrm{kg} / \mathrm{die}$ for 9 weeks \\
\hline
\end{tabular}

$K D$ Kawasaki disease, IVIG intravenous immunoglobulin, proBNP pro B-type natriuretic peptide NA Not available

intravenous (IV) therapy was started. Despite treatment, coronary dilatation increased. Anakinra $3 \mathrm{mg} / \mathrm{kg}$ was administered twice a day for 3 days, with immediate improvement of respiratory function and subsequent disappearance of the rash; clinical conditions and inflammation markers improved. However, a third echocardiograph showed diffuse dilatation of all coronaries, with the following $Z$ scores: left anterior descending $(\mathrm{LAD})=11$, right coronary artery $(\mathrm{RCA})=6.5$, and left main coronary artery $(\mathrm{LM})=5.6$. So, the patient also received infliximab $5 \mathrm{mg} / \mathrm{kg}$ and IV steroids, with clinical improvement. The patient received anakinra for 5 months at a dosage of $8 \mathrm{mg} / \mathrm{kg} / \mathrm{day}$ split in two doses. Echocardiography 8 months later showed an almost complete normalization of previous abnormalities ( $Z$ scores: $\mathrm{LAD}=0.84, \mathrm{RCA}=2.8$, and $\mathrm{LM}=1.6$ ).

The third case [22] described a 3-year-old girl with typical KD treated with IVIG $2 \mathrm{~g} / \mathrm{kg}$ and aspirin $100 \mathrm{mg} / \mathrm{kg}$. Anakinra $2 \mathrm{mg} / \mathrm{kg} /$ day was started on the 14th day from onset for persistent fever and increase of inflammatory markers despite two other doses of IVIG and two steroid boluses, with clinical and laboratory response within $24 \mathrm{~h}$.
Echocardiography was normal. Anakinra was administered for 2 weeks. After withdrawal, no relapses were observed and echocardiography remained normal.

In 2018, Guillaume et al. [23] reported on an 18-monthold boy with typical KD who received a first dose of IVIG on the sixth day of fever. On the seventh day, echocardiography revealed diffuse and fusiform aneurysms of all coronary arteries $[Z$ scores: $\mathrm{RCA}=6.89$, left coronary artery $(\mathrm{LCA})=6.06, \mathrm{LAD}=8.12$, and left circumflex $(\mathrm{LCX})=2.12$ ]. Fever subsided, but relapsed on the ninth day; therefore, IVIG was re-administered, along with three boluses of IV methylprednisolone. Despite therapy, on the 25 th day, the aneurysms worsened $(Z$ scores: $R C A=9.10$, $\mathrm{LCA}=7.47, \mathrm{LAD}=9.94$, and $\mathrm{LCX}=2.97)$. Anakinra $6 \mathrm{mg} /$ $\mathrm{kg} / \mathrm{day}$ was started for 10 weeks then tapered to $6 \mathrm{mg} / \mathrm{kg}$ every 2 days for 4 weeks and finally $6 \mathrm{mg} / \mathrm{kg}$ every 3 days for another 4 weeks up to withdrawal. Apyrexia was obtained in $24 \mathrm{~h}$ from anakinra injection, and C-reactive protein (CRP) normalized in 7 days; progressive reduction of aneurysm size allowed anticoagulation to be stopped after 4 months. Seven months from the onset of the disease, angio-CT showed a 
marked reduction in aneurysms $(Z$ scores: $R C A=2.06$, $\mathrm{LCA}=2.79, \mathrm{LAD}=4.34$, and $\mathrm{LCX}=1.47$ ).

Also in 2018, the effectiveness of anakinra was reported in a 16-year-old Caucasian boy with complete KD [24]. Echocardiography on the 12th day of fever revealed a slight dilatation of all coronary arteries; N-terminal pro B-type natriuretic peptide (NT-proBNP) blood levels were increased 2.5 -fold. The boy underwent a first IVIG infusion, without benefit; so, on the 15th day, he received anakinra at the dosage of $100 \mathrm{mg} /$ day. Resolution of fever and decrease of CRP and NT-proBNP occurred within $12 \mathrm{~h}$. Anti-IL-1 was continued for 4 weeks. Coronary angio-CT, which on the 19th day showed bulky aneurysms (diameters $0.8-0.9 \mathrm{~cm}$ ) and multiple stenosis of all coronaries throughout their course, was definitely improved at 6 months, with the disappearance of the stenosis and persistence of slight dilatation only in the proximal portion of the main vessels.

In 2018, Kone-Paut et al. [25] described the largest case series to date, reporting on 11 patients (eight males and three females), with an average age of 22 months (range 4 months-9 years), followed in seven European centers, affected by resistant KD, all successfully treated with anti-IL-1.

Nine of them had a complete form and two an incomplete form; two developed a KD shock syndrome and were transferred to intensive care unit. Seven patients had coronary anomalies on the first evaluation performed between the third and 12th day; three patients developed coronary artery abnormalities during the disease course; in two cases, myocarditis occurred, in one, there was evidence of valve dysfunction; only one patient had no cardiac complication. All patients had been treated with a first dose of IVIG at the standard dose of $2 \mathrm{~g} / \mathrm{kg}$ and aspirin $50 \mathrm{mg} / \mathrm{kg} /$ day and had received at least a second-line treatment. In particular, three patients had received anakinra after two ineffective treatments, four patients after three ineffective treatments, two patients after four ineffective treatments, and two other patients after five ineffective therapeutic attempts. The second line of treatment was a second IVIG infusion in five patients, infliximab in three patients, and methylprednisolone boluses in three other patients. As a third-line treatment, six patients received methylprednisolone, one oral prednisone, and one IVIG. Other drugs used (single patients) were cyclosporine, methotrexate, and infliximab.

All patients received anakinra at a dosage between 2 and $8 \mathrm{mg} / \mathrm{kg} / \mathrm{day}$, on average 25 days after onset (range 8-87 days) and for an average period of 15 days (range 6-87 days). The main reason for the use of anakinra was persistent fever (8/11), followed by gradual dilatation of the coronary arteries (7/11), persistence of clinical (2/11) or laboratory abnormalities (6/11), and severe myocarditis with KD shock syndrome in one case. The effectiveness of anakinra was assessed in terms of resolution of fever $(100 \%$ of cases), reduction of inflammation indexes (100\% of cases), and reduction of the $Z$ score (10/11 patients). A patient died suddenly, probably from a giant aneurysm rupture. The authors concluded by emphasizing the immediate clinical and laboratory response to anti-IL-1 (in most cases in $24 \mathrm{~h}$, and in one case, within 6 days), in particular in the case with KD shock syndrome (dramatically improved after the first injection of anakinra $6 \mathrm{mg} / \mathrm{kg}$ ).

In another retrospective series, involving $32 \mathrm{KD}$ patients, Flinn et al. [26] described a single patient with giant aneurysms, treated with several pharmacological treatments, and among them anakinra, but the authors did not specify the sequence nor the dose, nor the duration of the treatment. However, they did not report side effects.

Gamez-Gonzale et al. [27] also used anakinra in two patients with KD shock syndrome refractory to IVIG, but no further details on the clinical picture and outcome were provided.

In the last year, we found three other case reports on the use of anakinra in KD refractory to standard therapy.

Lind-Hoist et al. [28] reported on a 12-week-old infant with a history of vomiting for 10 days and irritability, who progressively developed respiratory distress, pericarditis, and severe dilatation of the coronary arteries ( $Z$ scores: LCA $=12.68$ and RCA $=9$ ). With the suspicion of atypical $\mathrm{KD}$, he was treated with IVIG, with only transient clinical improvement. Assuming a refractory form of $\mathrm{KD}$, infliximab was administered on the third day, followed by a second dose of IVIG and three boluses of methylprednisolone. On the 15th day, due to a considerable increase in ferritin (3349 $\mathrm{ng} / \mathrm{ml})$, triglycerides $(487 \mathrm{mg} / \mathrm{dl})$, and transaminases [alanine aminotransferase (ALT) $457 \mathrm{U} / 1$ ] and the detection of hemophagocytosis in bone marrow aspiration, the diagnosis of MAS was made. The child was treated with anakinra at increasing dosages (from 5 to $10 \mathrm{mg} / \mathrm{kg} /$ day), with gradual resolution of the clinical symptoms and normalization of laboratory tests. He was discharged on the 53rd day, on oral corticosteroids, aspirin, and anakinra, which were tapered and withdrawn over a period of 6 months.

Maggio et al. [29] described two siblings with parvovirus infection who subsequently developed two different clinical features of KD, one of whom was treated with anakinra. In particular, the case of interest concerns a 9-month-old girl who initially presented with fever, pharyngitis, conjunctivitis, vomiting, and diarrhea, with neutrophilic leukocytosis and severe anemia, initially attributed to parvovirus infection (positive IgM). However, on the 26th day, she developed coronary aneurysms ( $Z$ scores: $\mathrm{RCA}=6$ and LCA $=5.72$ ); therefore, the diagnosis of incomplete KD was made a posteriori. The patient received IVIG without improvement; so, anakinra was started at the dosage of $4 \mathrm{mg} / \mathrm{kg} /$ day, with progressive 
resolution of the cardiological findings up to normalization of the $Z$ score 25 days after the onset of anti-IL-1 therapy.

In the last case report, Gambacorta et al. [30] described a 9-month-old boy with classic KD. He received a first dose of IVIG on day 9, with benefit, and then, after clinical deterioration, a second dose of IVIG on day 14. On day 20, since the patient developed a new febrile peak and inflammatory markers increased, methylprednisolone pulses ( $30 \mathrm{mg} / \mathrm{kg}$ for 3 days) were administered, obtaining again a resolution of the symptoms. On day 25 , for the first time, the patient developed dilatation of the coronary arteries, with the appearance of small aneurysms involving the LCA, RCA, and the LAD. Despite a dose of infliximab $(5 \mathrm{mg} / \mathrm{kg}$ ) and the improved clinical conditions, the patient underwent serial echocardiograms that showed a progressive increase of the size of coronary arteries and eventually the development on day 37 of medium aneurysm of LCA ( $Z$ score 7.53) and giant aneurysms of RCA ( $Z$ score 11.49$)$ and $\operatorname{LAD}(Z$ score 11.2$)$. On day 40 , the infant presented with a new increase of inflammatory markers, edema of the hands and feet, and low-grade fever; so, anakinra $6 \mathrm{mg} / \mathrm{kg} /$ day was administered, and was followed by progressive clinical improvement with normalization of inflammatory markers and platelet count, and gradual reduction of the size of the coronary arteries and resolution of aneurysms.

In all these cases, the authors support the efficacy of anakinra in patients with refractory KD. In particular, anakinra was effective for severe and potentially fatal complications of KD, such as MAS [21, 28] and Kawasaki shock syndrome [20, 25, 27], but was also able to achieve the complete resolution of coronary dilatation and aneurysms or to prevent their worsening [20, 21, 23, 25, 26, 29, $30]$. In no case were side effects reported.

\subsection{Clinical Trials}

The first open-label, phase IIa clinical trial (KAWAKINRA, Eudract Number: 2014-002715-41, ClinicalTrials. gov NCT02390596) on anakinra in KD was performed in France and has been recently published [31].

The primary aim of the study was to determine the safety of blocking IL-1 in patients with KD unresponsive to IVIG. Sixteen patients were included, and they received subcutaneous anakinra at a starting dosage of 2 or $4 \mathrm{mg} /$ $\mathrm{kg} /$ day (depending on age and body weight), increased to $6 \mathrm{mg} / \mathrm{kg}$ if their temperature remained $>38^{\circ} \mathrm{C}$. Treatment duration was 14 days. The main outcome was abatement of fever; $75 \%$ of patients in the intention-to-treat group and $87.5 \%$ in the per-protocol group became afebrile within $48 \mathrm{~h}$ of the last escalation dose of anakinra. Disease activity measures, including physician's and parents' evaluations as well as CRP levels, improved in almost all cases.
At screening, 12 out of 16 patients had a coronary $Z$ score maximum of $>2$ and ten out of 16 had $>2.5$. At day 45 , five out of ten and six out of 12 patients reached a $Z$ score maximum of $<2.5$ and $<2$, respectively. Three patients had serious adverse events, which were resolved at the last evaluation. No severe infections occurred.

This study supports the early use of anakinra in cases refractory to IVIG, demonstrating that it is quickly effective on KD symptoms, inflammation parameters, and dilatation of the coronary arteries in most patients, with good tolerability.

The ANAKID trial (ClinicalTrials.gov NCT2179853) [32], an American phase I/IIa dose-escalation study whose primary objective is to assess the safety and tolerability of anakinra in the acute phase of KD in patients not responsive to a first cycle of IVIG and aspirin, is currently underway. The secondary objective of the trial is to evaluate the pharmacokinetics of the drug.

Eligibility criteria are the following: American Heart Association criteria for $\mathrm{KD}$, age $>1$ month, and $Z$ score $\geq 3$ within 20 days of onset. The treatment schedule consists of at least 2 weeks of therapy, with the first two doses administered intravenously ( $4 \mathrm{mg} / \mathrm{kg} /$ day $)$. Patients with a $Z$ score $\geq 2.5$ or an aneurysm after 2 weeks will complete 6 weeks of therapy. We are awaiting conclusion of the study and publication of full results.

Another study (ANACOMP, a randomized, phase III, multicenter trial comparing the efficacy and safety of anakinra versus IVIG retreatment in patients with KD who failed to respond to initial standard IVIG treatment) has been planned and approved, and will start soon in France.

\section{Conclusion}

To date, guidelines for the treatment of KD resistant cases are not based on strong evidence, and sometimes a second infusion of IVIG is used in clinical practice. However, this strategy has not yet been proven to be effective in a prospective controlled trial, and new treatments are now available. Of note, a new disorder possibly linked to SARSCoV-2 infection has been described both in Europe and in the USA; it has been termed pediatric multi-inflammatory syndrome temporally associated with COVID-19 (PIMSTS) or multisystem inflammatory syndrome associated with coronavirus disease 2019 (MIS-C). This condition shares features with KD, i.e., fever, rash, and mucositis, but also has several differences, such as older age at onset and a high prevalence of myocarditis and gastrointestinal involvement. Case series have been published. Also, on the basis of previous experience in adults with COVID-19 [33] anakinra has been used in resistant cases [34, 35] even 
though a lack of controlled trials in this setting does not allow firm conclusions yet.

In this review, we have summarized the preclinical studies related to IL-1 involvement in the pathogenesis of $\mathrm{KD}$ and the clinical experience regarding anakinra treatment for this disorder published so far. The clinical cases described show that anakinra has been used in most cases after at least two ineffective treatments, in cases of coronary aneurysms already at diagnosis, and in cases of major complications of KD such as MAS or KD shock syndrome, without relevant side effects. With regard to cost, in Italy, using a mere economic comparison, the cost of a second IVIG dose for refractory patients as a therapeutic alternative to a 4-week cycle of anakinra is more expensive, up to $80 \%$ more (counting only drug costs).

While awaiting the results of the trials currently finished and of those which are ongoing or planned, we acknowledge the possible role of anakinra for KD patients who do not respond to a first IVIG infusion.

\section{Declarations}

Funding We received no funding for this article.

Conflict of interest There are no conflicts of interest. There are no competing interests.

Ethics approval Not applicable.

Consent to participate Not applicable.

Consent for publication Not applicable.

Availability of data and material Not applicable.

Code availability Not applicable.

Author contributions All authors contributed to the planning and writing of the manuscript.

\section{References}

1. McCrindle BW, Rowley AH, Newburger JW, et al. Diagnosis, treatment, and long-term management of Kawasaki disease: a scientific statement for health professionals from the American Heart Association. Circulation. 2017;135:e927-99.

2. Phuong LK, Curtis N, Gowdie P, et al. Treatment options for resistant Kawasaki disease. Pediatr Drugs. 2018;20:59-80.

3. Dusser P, Koné-Paut I. IL-1 Inhibition may have an important role in treating refractory Kawasaki disease. Front Pharmacol. 2017;8:163.

4. Sims JE, Smith DE. Regulation of interleukin-1 activity is enhanced by cooperation between the interleukin- 1 receptor type II and interleukin-1 receptor accessory protein. Eur Cytokine Netw. 2003;14:77-81.
5. Ramírez J, Canete JD. Anakinra for the treatment of rheumatoid arthritis: a safety evaluation. Expert Opin Drug Saf. 2018; 17:727-732.

6. Haddad YB, Robert S, Salers P, et al. Sterile inflammation of endothelial cell-derived apoptotic bodies is mediated by interleukin-1 $\alpha$. Proc Natl Acad Sci USA. 2011;108:20684-9.

7. Bettiol A, Lopalco G, Emmi G, et al. Unveiling the efficacy, safety, and tolerability of anti-interleukin-1 treatment in monogenic and multifactorial autoinflammatory diseases. Int J Mol Sci. 2019;20:1898.

8. McDermott MF. Rilonacept in the treatment of chronic inflammatory disorders. Drugs Today (Barc). 2009;45:423-30.

9. Chakraborty A, Tannenbaum S, Rordorf C, et al. Pharmacokinetic and pharmacodynamic properties of canakinumab, a human anti-interleukin-1 $\beta$ monoclonal antibody. Clin Pharmacokinet. 2012;51:e1-e1.

10. Leung DY, Cotran RS, Kurt-Jones E, et al. Endothelial cell activation and high interleukin-1 secretion in the pathogenesis of Kawasaki disease. Lancet. 1989;2:1298-302.

11. Alphonse MP, Duong T, Shumitzu C, et al. Inositol-triphosphate 3-kinase $\mathrm{C}$ mediates inflammasome activation and treatment response in Kawasaki disease. J. Immunol. 2016;197:3481-9.

12. Weng KP, Hsieh KS, Ho TY, et al. IL-1B polymorphism in association with initial intravenous immunoglobulin treatment failure in Taiwanese children with Kawasaki disease. Circ J. 2010;74:544-51.

13. Fury W, Tremoulet AH, Watson VE, et al. Transcript abundance patterns in Kawasaki disease patients with intravenous immunoglobulin resistance. Hum Immunol. 2010;71:865-73.

14. Hoang LT, Shimizu C, Ling L, et al. Global gene expression profiling identifies new therapeutic targets in acute Kawasaki disease. Genome Med. 2014;6:541.

15. Lehman TJ, Walker SM, Mahnovski V, et al. Coronary arteritis in mice following the systemic injection of group B Lactobacillus casei cell walls in aqueous suspension. Arthritis Rheum. 1985;28:652-9.

16. Lee YH, Schulte DJ, Shimada K, et al. Il-1 $\beta$ is crucial for induction of coronary artery inflammation in a mouse model of Kawasaki disease. Circulation. 2012;125:1542-50.

17. Wakita D, Kurashima Y, Crother TR, et al. Role of interleukin-1 signaling in a mouse model of abdominal aortic aneurysm associated with Kawasaki Disease. Arterioscler Thromb Vasc Biol. 2016;36:886-97.

18. Gorelik M, Lee Y, Abe M, et al. The IL-1 receptor antagonist, anakinra, prevents myocardial dysfunction in a mouse model of vasculitis and myocarditis of Kawasaki disease. Clin Exp Immunol. 2019;198:101-10.

19. Noval Rivas M, Wakita D, Franklin MK et al. Intestinal permeability and IgA provoke immune vasculitis linked to cardiovascular inflammation. Immunity. 2019;51:508-521e6.

20. Cohen S, Tacke CE, Straver B, et al. A child with severe relapsing Kawasaki disease rescued by IL-1 receptor blockade and extracorporeal membrane oxygenation. Ann Rheum Dis. 2012;71:2059-61.

21. Shafferman A, Birmingham JD, Cron RQ. High-dose anakinra for the treatment of severe neonatal Kawasaki disease: a case report. Pediatr Rheumatol. 2014;12:26.

22. Sánchez-Manubens J, Gelman A, Franch N, et al. A child with resistant Kawasaki disease successfully treated with anakinra: a case report. BMC Pediatr. 2017;17:102.

23. Guillaume M-P, Reumaux H, Dubos F. Usefulness and safety of Anakinra in refractory Kawasaki disease complicated by coronary artery aneurysm. Cardiol Young. 2018;28:739-42.

24. Blonz G, Lacroix S, Benbrik N, et al. Severe late-onset kawasaki disease successfully treated with anakinra [published online 
ahead of print, 2018 Jun 15]. J Clin Rheumatol. 2018. https://doi. org/10.1097/rhu.0000000000000814.

25. Kone-Paut I, Cimaz R, Herberg J, et al. The use of interleukin 1 receptor antagonist (Anakinra) in Kawasaki disease: a retrospective cases series. Autoimmunity Rev. 2018;17:768-74.

26. Flinn AM, Gavin PJ, McMahon CJ, et al. Kawasaki disease-a review of treatment and outcomes in an Irish pediatric cohort 2010-14. Irish Med J. 2018;111:692.

27. Gamez-Gonzale LB, Quintero IM, Castolo MC, et al. Kawasaki disease shock syndrome: a unique and serious subtype of Kawasaki disease. Pediatr Int. 2018;60:781-90.

28. Lind-Host M, Hartling UB, Christensen AE. High-dose anakinra as treatment for macrophage activation syndrome caused by refractory Kawasaki disease in an infant. BMJ Case Report. 2019;12:e229708.

29. Maggio MC, Cimaz R, Alaimo A, et al. Kawasaki disease triggered by parvovirus infection: an atypical case report of two siblings. J Med Case Rep. 2019;13:104.

30. Gambacorta A, Buonsenso D, De Rosa G, et al. Resolution of giant coronary aneurisms in a child with refractory Kawasaki disease treated with anakinra. Front Pediatr. 2020;8:195.

31. Kone-Paut I, Tellier S, Belot A, et al. Open label, phase II study with anakinra in intravenous immunoglobulin-resistant Kawasaki Disease. Arthritis Rheumatol. 2020. https://doi.org/10.1002/ art.41481. Online ahead of print.
32. Tremoulet AH, Jain S, Kim S, et al. Motivation and study design for a phase I/IIa study of anakinra in children with Kawasaki disease and early coronary artery anomalies (ANAKID study). Contemp Clin Trials. 2016;48:70-5.

33. Navarro-Millán I, Sattui SE, Lakhanpal A, et al. Use of anakinra to prevent mechanical ventilation in severe COVID-19: a case series. Arthritis Rheumatol. 2020. https://doi.org/10.1002/ art.41422.

34. Davies P, Evans C, Kanthimathinathan HK, et al. Intensive care admissions of children with paediatric inflammatory multisystem syndrome temporally associated with SARS-CoV-2 (PIMSTS) in the UK: a multicentre observational study. Lancet Child Adolesc Health. 2020;S2352-4642(20):30215-7. https://doi. org/10.1016/S2352-4642(20)30215-7.

35. Feldstein LR, Rose EB, Horwitz SM, et al. Multisystem inflammatory syndrome in U.S. children and adolescents. N Engl J Med. 2020; published online June 29. https://doi.org/10.1056/nejmo a202168. 